\title{
Il simbolico e la via del movimento delle donne
}

\author{
di Chiara Zamboni
}

chiara.zamboni@univr.it

\begin{abstract}
Symbolic politics has been one of the key phrases in Italian feminism since the 1970s. This text describes the historical origins and significance of this concept together with the difference in the use of the symbolic dimension in traditional male politics. The most important difference is that female politics are born from living and affective relations which cannot be permanently established and rotate around non-formalized procedural practices. The sense that emerges is felt during the actual process and the symbolic is therefore experienced and transformed from the inside with the authority that comes from these practices, which influence and modify each other due to their closeness. They spread by proximity.
\end{abstract}

Keywords: Symbolic politics, Italian Feminism, The Sexual Difference, Practices

\section{Introduzione}

Desidero portare un contributo al tema che riguarda il legame tra la politica e il simbolico, partendo dalla via tracciata dal movimento delle donne, che più volte ha mostrato una particolare attenzione al simbolico ${ }^{1}$. Quella delle donne è una politica che ha una sua storia e forme significanti. Innanzitutto non è affidata alla rappresentanza né ai partiti, dunque si scosta dalla democrazia nelle forme istituite che conosciamo. Un criterio che la caratterizza è che l'organizzazione viene evitata a favore di relazioni fluide e allo stesso tempo efficaci. Si può dire che sono proprio le relazioni a creare il tessuto portante di ogni espressione di questa politica. Con tutti i vantaggi e gli svantaggi che ciò comporta, perché nelle relazioni circola qualcosa di vitale e orientante, ma le relazioni tra donne, non essendo istituite, possono

\footnotetext{
${ }^{1}$ Imprescindibile per capire la politica del simbolico il testo di Ida Dominijanni, "Politica del simbolico e mutamento", IG Informazioni, III, 1992, pp. 131-157.
}

Materiali di Estetica - N. 8.2: 2021, Pagina 279 
affievolirsi, precipitare in conflitti insormontabili, venir meno. Il che significa che il movimento è come un'onda che ha momenti di forte intensità e momenti in cui sembra quasi scomparire.

È un movimento trasversale ai partiti, ai luoghi di lavoro, alle diverse organizzazioni pubbliche, e questo lo si vede ad esempio in certi legami imprevisti tra le donne del parlamento - indipendentemente dalla collocazione politica - rispetto a quelle questioni in cui ne va di mezzo la libertà femminile.

È bene subito sgombrare il campo da un'idea fuorviante. Potrebbe infatti sembrare che parlare della centralità del simbolico nella politica delle donne nasca dall'esigenza di aggiungere una prospettiva ad altre, come una parte di un tutto che il tema suggerisce. Non è così. La politica delle donne non è una delle possibili visioni semplicemente alternative. Questo significherebbe che la politica è legata ad una storia maschile che risulta paradigma onnicomprensivo, rispetto al quale quella delle donne sarebbe un'aggiunta importante, senz'altro, ma semplicemente da includere come portatrice di una specificità interessante ma minoritaria.

Per spiegare, si può partire dal fatto che al centro di questa politica sono le pratiche, iniziate da donne, che nascono e variano nel tempo creando effetti trasformativi sia su di sé sia nel contesto. Pratiche fondate su relazioni in prima persona e in divenire. In questo senso è una politica incommensurabile rispetto al pensiero politico maschile per quello che si è dato storicamente.

È importante puntualizzare che in questo testo vengono adoperati i termini politica delle donne e movimento delle donne con un'ampiezza semantica maggiore di movimento femminista o semplicemente femminismo. Molte donne hanno riconosciuto come vincolanti e pubbliche alcune relazioni con altre donne senza per questo dichiararsi femministe. Il femminismo - a partire dalle sue varie declinazioni interne per cui si parla anche di femminismi - ha comunque offerto espressione simbolica a questi legami e a queste azioni pubbliche, dando ad essi un'impronta consapevolmente politica. 
Emblematico in questi anni il movimento del \#metoo, in cui si sono esposte e hanno preso la parola donne che non necessariamente si sono riconosciute come femministe ${ }^{2}$. In questo senso il movimento politico delle donne è più diffuso e fluido di quanto non siano i gruppi femministi.

\section{Pratiche e simbolico}

Per parlare del simbolico femminista non ricostruirò i tanti decenni di femminismo italiano, per i quali rimando ai diversi testi che ne trattano ${ }^{3}$, ma mi concentrerò sul pensiero politico nato nel percorso sperimentale di queste pratiche ${ }^{4}$.

Prima però ho bisogno di dare alcune coordinate del dibattito sul simbolico in area filosofica e psicoanalitica per coglierne le linee essenziali e misurare poi la differenza rispetto al pensiero femminista. Lacan nel libro terzo dei seminari descrive il linguaggio simbolico come una città con strade centrali -

\footnotetext{
2 Cfr. Maria Chiara Risoldi, \#Me Too: il patriarcato dalle mimose all'hashtag, Antonio Trombolini Editore, Loreto 2018.

3 Vedi innanzitutto Libreria delle donne di Milano, Non credere di avere dei diritti. La generazione della libertà femminile nell'idea e nelle vicende di un gruppo di donne, Rosenberg\&Sellier, Torino 1989; Diotima, Il pensiero della differenza sessuale, La Tartaruga, Milano 1987; Lia Cigarini, La politica del desiderio, Pratiche Editrice, Parma 1995; Adriana Cavarero, Nonostante Platone, Editori Riuniti, Roma 1990; Luisa Muraro, L'ordine simbolico della madre, Editori Riuniti, Roma 1991; Lea Melandri, Una visceralità indicibile. La pratica dell'inconscio nel movimento delle donne egli anni Settanta, FrancoAngeli, Milano 2000; Teresa Bertilotti, Anna Scattigno, Il femminismo degli anni Settanta, Viella, Roma 2005; Marisa Forcina, Rappresentazioni politiche della differenza, FrancoAngeli, Milano 2009; Marina Santini, Luciana Tavernini, Mia madre femminista. Voci da una rivoluzione che continua, Il Poligrafo, Padova 2015; Tristana Dini, La materiale vita. Biopolitica, vita sacra, differenza sessuale, Mimesis, Milano 2016; Diotima, Femminismo fuori sesto. Un movimento che non può fermarsi, Liguori, Napoli 2017.

${ }^{4}$ Per il dibattito contemporaneo sul femminismo e il pensiero della differenza rimando a Linda Zerilli, Femminism and the Abyss of Freedom, University of Chicago Press, Chicago 2005; Geneviève Fraisse, Il mondo è sessuato. Femminismo e altre sovversioni (2016), tr. it. a cura di Annarosa Buttarelli, Nottetempo, Milano 2019; Anne Emmanuelle Berger, And Yet she speaks! "Italian Feminism" and Language, in Cesare Casarino, Andrea Righi (ed. by), Another Mother: Diotima and the Symbolic Order of Italian Feminism, University of Minnesota Press, Minneapolis 2018; Françoise Duroux, Il paradigma perturbante della differenza sessuale. Una filosofia femminista, tr. it. a cura di Stefania Tarantino e Chiara Zamboni, Mimesis, Milano 2021.; Danila Cannamela, "I am an atypical mother': Motherhood and maternal language in Giovanna Cristina Vivinetto's poetry”, Forum Italicum, LV/1, 2021, pp. 85-108.
} 
mi immagino come il cardo e il decumano nelle città romane - che sono punto di riferimento di vie che da esse si dipartono. Che ne sarebbe della civiltà medievale se dal centro della città venisse meno il nome di Dio? Le maglie di un'intera civiltà si disferebbero. Allo stesso modo nella civiltà patriarcale il Nome-del-Padre è il significante che regge simbolicamente un ordine sociale. Lacan adopera l'immagine dei punti di capitone che reggono i materassi, quando i materassi sono con la lana. Sono quelle impunture fatte con aghi molto grossi che collegano un lato e l'altro del materasso con fili molto forti. Questo per impedire che la lana vada di qua e di là in forma disordinata, caotica. I punti di capitone sono i significanti portanti di una civiltà.

Il significante Nome-del-Padre è simbolico per Lacan nel senso che non si tratta semplicemente di un padre concreto che governa una famiglia né di un padre che si immagina di avere potere sulla famiglia, bensì di un padre che accetta di esercitare la funzione di padre senza identificarsi in essa. In altre parole, di essere un servitore della funzione, senza trarne un godimento per sé. La funzione è incarnata nel singolo uomo che l'assume, ma è simbolica perché ha un significato che non coincide con il singolo che l'incarna. Ed è in quanto simbolica che istituisce un ordine sociale ${ }^{5}$.

Consideriamo a questo punto il contributo di Michel Foucault alla questione del simbolico. Mi spiego con un testo particolarmente preciso in questo senso che è L'ordine del discorso. Il discorso viene visto non solo per quello che permette di dire, ma soprattutto per il sistema di esclusione sul quale si regge e cioè l'interdizione alla parola (alcuni possono prendere la parola in modo che sia ascoltata e onorata e altri no), la marginalizzazione della follia (il discorso del folle semplicemente non è preso in considerazione),

\footnotetext{
${ }^{5}$ Vedi Jacques Lacan, La psicosi. Seminario terzo, tr. it. a cura di Giacomo Contri, Einaudi, Torino 1985, capitoli XXI-XXIII, pp. 307-348. Su questi passaggi di Lacan che riguardano il simbolico si può leggere: Felice Cimatti, Il taglio. Linguaggio e pulsione di morte, Quodlibet, Macerata 2015; Massimo Recalcati, Jacques Lacan. Desiderio, godimento e soggettivazione, Cortina, Milano 2012, in particolare cap. 2 ("La dialettica del simbolico") e cap. 3 ("Nomi del padre?"). Sul rapporto tra Lacan e la questione della sessualità si veda Alenka Zupančič, Che cos’è il sesso? (2017), tr. it. di Pietro Bianchi, Ponte alle Grazie, Milano 2018.
} 
e per finire la volontà di verità, che definisce i criteri di vero e falso ${ }^{6}$. Ciò che rende simbolico il discorso è una molteplicità di pratiche rituali, cioè pratiche ripetute nella loro forma e sostenute da un potere diffuso, pratiche di assoggettamento. Si pensi ad esempio all'insegnamento che in questa prospettiva viene visto come una ritualizzazione della parola che stabilisce chi ha la qualifica di parlare e chi no e dunque assegna ruoli differenziati sia per quanto riguarda il potere che per il sapere ${ }^{7}$. Non si tratta per Foucault di pratiche come processi creativi come nella politica delle donne, ma di pratiche rituali che implicano l'interpretazione di vere e proprie tecniche di comportamento in cui sono chiare le dipendenze da poteri diffusi.

Attraversando queste due teorie - lacaniana e foucaultiana - si può pensare che la questione politica del simbolico si collochi su questo livello del linguaggio, che distribuisce posizioni rituali ripetitive ${ }^{8}$. E che dunque l'azione politica stia nel modificare l'ordine del discorso, distribuendo diversamente le pratiche di potere e sapere.

In realtà il movimento delle donne non ha optato solo per la trasformazione delle pratiche discorsive, perché, se si fosse limitato a questo, sarebbe rimasto nello stesso paradigma di potere e di inclusione-esclusione. Il movimento ha fatto qualcosa di diverso non per presa di posizione teorica, ma

\footnotetext{
${ }^{6}$ Vedi Michel Foucault, L'ordine del discorso (1971), tr. it. di Alessandro Fontana, Einaudi, Torino 1972, p. 17, dove Foucault sintetizza i punti centrali dei processi di esclusione, che ha spiegato nei capitoli precedenti. Sul legame e la differenza tra Lacan e Foucault riguardo al discorso si legga: Andrea Muni, "Foucault e Lacan. L'amicizia, il discorso, il soggetto etico", aut aut, CCCLXXXVII, 2020, pp. 69-86. Su Foucault e la questione del potere si legga Lorenzo Bernini, Le pecore e il pastore: Critica, politica, etica nel pensiero di Michel Foucault, Liguori, Napoli 2008. Sul tema del linguaggio in Foucault, si leggano i due fondamentali testi di Deleuze: sia Gilles Deleuze, Il sapere. Corso su Michel Foucault (1985-1986), vol. I, tr. it. di Lorenzo Feltrin, Ombre Corte, Verona 2014, come anche Gilles Deleuze, Foucault (1986), tr. it. di Filippo Domenicali, Orthotes, Napoli 2018. Alcune riflessioni sul rapporto tra il pensiero di Michel Foucault e quello femminista si legga Salvo Vaccaro, "Lo sguardo e la differenza. Michel Foucault e il pensiero delle donne", in Salvo Vaccaro, Mario Coglitore (a cura di), Michel Foucault e il divenire donna, Mimesis, Milano 1997, pp. 17-45.

7 Vedi sull'insegnamento come procedura di assoggettamento del discorso M. Foucault, L'ordine del discorso, cit., p. 35.

${ }^{8}$ Si sa che Lacan non prevede un'azione politica che trasformi i significanti che strutturano l'ordine simbolico. Sto accennando in questo testo a delle conseguenze che si possono trarre dalla sua concezione di simbolico.
} 
fondamentalmente perché il desiderio non era quello del governo della realtà, ma di aprire spazi di libertà nel presente a partire da pratiche, di cui fossero soggetti le donne e in prospettiva donne e uomini assieme. Una conseguenza essenziale di queste pratiche è stata quella di entrare in conflitto sul piano del linguaggio contro le configurazioni del simbolico dominante, ma avendo radici in un humus di forme di vita che si sono andate via via creando.

Per questo è proprio il passo d'entrata ad essere diverso. È come si entra nella questione del simbolico che fa la differenza. Tutto si gioca su un modo diverso di intendere le pratiche. Nella prospettiva del governo della realtà le pratiche di comportamento proposte sono descrittive e normative allo stesso tempo. Lo spazio di interpretazione delle pratiche è minimo: ogni interpretazione della norma è una variazione possibile, che però rimane all'interno del campo proposto. Invece ciò che caratterizza le pratiche nel movimento politico delle donne è che si tratta di processi avviati con altre donne con un minimo di regole iniziali, ma lasciando che queste si trasformino con l'andamento che prende l'esperienza interna al processo. È il desiderio soggettivo che muove. Perciò questi processi possono cambiare profondamente o venire meno. Si tratta di percorsi di sperimentazione, di scoperta, caratterizzati dall'imparare dall'esperienza ${ }^{9}$.

Porto come primo esempio la pratica dell'autocoscienza: una delle più note e diffuse del femminismo e ripresa anche oggi in forme diverse, ma fedeli allo spirito iniziale. È nata negli Stati Uniti alla fine degli anni Sessanta come forme di presa di coscienza di collettivi di donne e ha rapidamente preso piede in Italia. Sostanzialmente nasce dal bisogno di trovarsi tra donne come atto politico di sottrazione allo sguardo, al discorso e all'immaginario maschile; atto fondato sulla fiducia nei confronti delle altre per individuare assieme non solo le forme dell'oppressione patriarcale ma soprattutto bisogni e desideri

\footnotetext{
$9 \mathrm{Si}$ veda sulla sperimentazione nelle pratiche come passaggio necessario al simbolico Libreria delle donne di Milano, Non credere di avere dei diritti, cit., pp. 56-57.
} 
soggettivi che, con la mediazione delle altre donne, trovano così autorizzazione ad esprimersi. Dunque nasce dal desiderio di una parola autonoma di verità su quello che si vive in prima persona, sapendo che ha un valore politico, dunque non solo personale, perché condiviso e mosso dal desiderio di un nuovo modo di vivere in comune.

Mi sembrano due i passaggi simbolici di questa pratica. Il primo è la scommessa sul fatto che ciò che si legge di vero nell'esperienza che si vive abbia un valore di verità per altre e altri. Non perché altri l'abbiano vissuta, ma in quanto ciò che si coglie è portatore di un significato non solo soggettivo ma prospettico di un contesto. Di un mondo. Uscire dal soggettivismo, pur parlando della esperienza vissuta in prima persona, si sostanzia nell'entrare nel linguaggio condiviso in cui sono in corso conflitti sul modo di intendere la realtà. Viene portato ciò che si è guadagnato di verità e inevitabilmente ciò comporta una critica rispetto ad altre letture della realtà. L'espressione di una verità così guadagnata va di pari passo con la decostruzione di altre posizioni in campo. Questo è uno degli aspetti del valore simbolico e politico di questo processo.

Il secondo aspetto simbolico dell'autocoscienza si fonda sul fatto che è una pratica fondata su racconti ${ }^{10}$. L'esperienza vissuta soggettivamente viene detta alle altre del piccolo gruppo. È un racconto che parte dal presupposto di dire qualcosa di vero del presente e del passato. Penso ad esempio al diario di Carla Lonzi, che ha introdotto il termine di autocoscienza. Penso al suo desiderio di essere autentica, di dire la verità dei legami con le compagne del piccolo gruppo di Rivolta femminile, che rimanda per risonanza alle relazioni con la madre e con le sorelle, con il padre, ai vissuti a scuola dalle suore ${ }^{11}$. Nel

\footnotetext{
${ }_{10}$ Sull'esperienza femminile e la narrazione si legga Adriana Cavarero, Tu che mi guardi, tu che mi racconti. Filosofia della narrazione, Feltrinelli, Milano 1997. Più estesamente sull'importanza del racconto per la politica: Olivia Guaraldo, Politica e racconto, Meltemi, Roma 2003.

11 Mi riferisco a Carla Lonzi, Taci, anzi parla. Diario di una femminista, Ed. Rivolta femminile, Milano 1978. Sulla famiglia vedi ad esempio pp. 17-25 e pp. 90-93, sul convento
} 
racconto l'attenzione è rivolta al presente patito e costantemente confrontato con il passato, al trovare in questo dei legami e fare scoperte che aprono la coscienza.

Introduco l'interpretazione che ne ha dato Manuela Fraire, una psicoanalista femminista che ha partecipato ai gruppi di autocoscienza. Lei considera l'azione dell'inconscio in questi racconti. Si discosta dall'interpretazione più nota di Freud, per cui possono emergere nel racconto elementi rimossi del passato e si appoggia invece alla teoria dell'après coup, per la quale fa riferimento ad altre parti del pensiero di Freud. Si tratta di esperienze vissute, ma non pensate, dunque non rimosse. L’invenzione linguistica - l'après coup - le crea nel presente come avvenimenti del passato. Sono molto lontane dal concetto di proiezione. Scrive Manuela Fraire:

L'après coup si vale dunque di costruzioni e per questo motivo ha costituito l'aspetto forse più rivoluzionario del pensiero freudiano, poiché non è stato concepito come una retroazione del presente sul passato bensì come costruzione nel presente di ciò che non accadde, un vissuto non pensato, nel passato. Questo modo di intendere il lavoro che la memoria fa sul ricordo è compatibile col lavoro che il femminismo ha fatto attraverso la pratica dell'autocoscienza ogni volta che si è smarcato dal bisogno di mostrare la fondatezza storica del discorso delle donne, ogni volta cioè che ha proceduto alla ricerca del senso che ogni ricostruzione fedele solo ai fatti impedisce ${ }^{12}$.

È una pratica che è simile all'invenzione del simbolo zero nel momento in cui è stato introdotto nella matematica europea nel medioevo, sulla scorta della matematica araba. Una volta posto nella serie dei numeri, crea nel presente qualcosa che sembra come esistito da sempre se pure in forma abbozzata, non espresso ma implicitamente usato nel calcolo dei matematici antichi.

pp. 28-32, sul gruppo di donne e il riconoscimento pp. 72-84. Naturalmente ho indicato solo alcuni percorsi all'interno di un testo di milletrecento pagine.

12 Manuela Fraire, "Attualità e inattualità dell'autocoscienza", in Chiara Zamboni (a cura di), La carta coperta. L'inconscio nelle pratiche femministe, Moretti\&Vitali, Bergamo 2019, pp. 54-60, qui p. 58 . 
L'après-coup dunque è una creazione, sì, ma non arbitraria. Si è di fronte a vissuti non pensati, non legati al ricordo di fatti del passato. I racconti delle donne dei piccoli gruppi di autocoscienza creano reale, esprimendo percorsi di senso, ma sulla base di qualcosa di vissuto non pensato, che resta nell'area dell'indeterminato. Del non referenziale. È questa la forza simbolica delle narrazioni in questa pratica orientata al senso del reale più che alla fedeltà ai fatti.

Nel dibattito femminista contemporaneo la pratica dell'autocoscienza è stata ripresa e rilanciata da quella del partire da sé, cioè dalla storia dell'esperienza di cui si è soggettivamente testimoni letta in modo da avere una chiave di interpretazione della realtà contemporanea. Vi sono transitati gli aspetti più interessanti, che hanno caratterizzato l'autocoscienza.

Il secondo esempio di pratica femminista, che mostro per la sua portata simbolica, fa riferimento all'autorità. In alcuni testi femministi come Non credere di avere dei diritti della Libreria delle donne di Milano viene ricostruita la genesi di questo concetto nelle esperienze vissute in gruppi di donne. Viene raccontato come una scoperta il fatto per cui la parola di alcune donne veniva ascoltata più di quella di altre, ripresa e rilanciata. Con questo si coglieva una differenza, che però rappresentava una differenza generatrice di potenzialità per le altre ${ }^{13}$. Anche in questa narrazione agisce quello che abbiamo già visto come un après-coup. Nel senso che nel momento in cui si è scoperto l'agire simbolico dell'autorità, immediatamente lo si è percepito come qualcosa che c'era sempre stato tra donne, però nella forma di un vissuto non conosciuto.

L'autorità è un concetto relazionale non solo perché per avere efficacia ha bisogno del riconoscimento altrui, ma anche perché è fondamentalmente mediatore: è generatore di potenza d'essere per altri. In questo senso è

13 Vedi Libreria delle donne di Milano, Non credere di avere dei diritti, cit., p. 87 e pp. 162 163. Sul tema dell'autorità in chiave teorico-femminista si legga anche Diotima, Oltre l’uguaglianza. Le radici femminile dell'autorità, Liguori, Napoli 1995. 
trasformativo e fa da ponte verso dimensioni di vita più ricche. Lo possiamo trovare facilmente nella nostra esperienza, anche quando non ne abbiamo avuto agli inizi consapevolezza. Ad esempio, nei nostri anni di scuola, è probabile che molti di noi si ricordino di qualche docente che è stata o stato fondamentale per alcune lezioni indimenticabili o per un certo modo di rapportarsi alla cultura, la lingua, i testi, la politica. Alla vita. Sentire la sua autorità e seguire la via che indicava si sono intrecciati l'uno all'altro, ma questo perché riusciva a toccare il nostro movimento desiderante ${ }^{14}$. Il femminismo ha mostrato come ciò rimandi all'autorità implicita esercitata dalla madre sulla figlia o figlio durante l'infanzia. Anche se è bene ricordare che nel caso della madre la dipendenza è stata molto più forte e la contrattazione più necessaria, mentre nel caso degli insegnanti il sentirne l'autorità nasce da una dipendenza legata ad un desiderio libero. Eppure molto del legame con la madre c'è nel riconoscimento di autorità, la fiducia ad esempio $^{15}$.

È un concetto mediatore e relazionale e in questo senso ci porta ad un'altra sfaccettatura del simbolico che qui si significa principalmente come elemento trasformatore.

Averlo introdotto non è stata un'azione ingenua. Le donne che per prime ne hanno parlato erano consapevoli del fatto che il Novecento è stato un secolo nel quale la parola autorità ha avuto una storia non facile. Dall'idea di stato autoritario nel periodo fascista alla dittatura nazista che ha minato alla base l'autorità paterna nella società civile, alla critica alla famiglia autoritaria da parte dei movimenti libertari degli anni Settanta fino all'attacco all'autorità patriarcale da parte del femminismo stesso. Dunque non si è trattato di una

\footnotetext{
${ }^{14}$ Un'analisi molto fine della capacità di suscitare un movimento desiderante da parte di una insegnante si trova in Massimo Recalcati, L'ora di lezione. Per un'erotica dell'insegnamento, Einaudi, Torino 2014. Vedi anche alcune pagine dedicate a Formaggio come professore di liceo in Gabriele Scaramuzza, "Per Dino Formaggio", in Gabriele Scaramuzza, Smarrimento e scrittura, Mimesis, Milano 2019, pp. 117-118.

${ }^{15}$ Sul legame tra la mediazione simbolica dell'autorità e il primo rapporto con la madre si veda Luisa Muraro, Autorità, Rosenberg\&Sellier, Torino 2013, p. 100 e anche pp. 83-84.
} 
strada facile anche perché il tramonto stesso dell'idea di autorità nel Novecento ha dato spazio al concetto di potere come unico interpretante dei contesti politici e sociali. Da parte del femminismo non ha significato recuperare un'antica idea di autorità di origine paterna, ma di esprimere in una figura simbolica l'aver sperimentato la capacità generativa di alcuni rapporti tra donne in analogia con il primo rapporto di fiducia con la madre e il riconoscimento reciproco di questo. Ed è bene aggiungere che anche gli uomini hanno la percezione di questa autorità di matrice materna, perché l'hanno sperimentata, ma in un modo diverso dalle donne. Partono dalla loro differenza dalla madre ${ }^{16}$.

Per questo è anche molto diverso dal modo in cui Hannah Arendt affronta il tema dell'autorità in quel saggio fondamentale che è Che cos'è l'autorità? e questo per diversi motivi. Innanzitutto perché Arendt si confronta soltanto con la storia maschile; in secondo luogo perché considera l'autorità che si incontra nell'insegnamento e nella pedagogia come prepolitica mentre il movimento delle donne la considera politica, ma soprattutto perché lei la ritiene definitivamente tramontata ${ }^{17}$. E tuttavia il suo saggio rimane un riferimento sia per quel che ne dice, sia per aver notato come la scomparsa dell'autorità - di matrice maschile - abbia lasciato spazio solo al potere e infine per l'osservazione illuminante che il venir meno dell'autorità va di pari passo con il venir meno della libertà ${ }^{18}$. È proprio questo il punto anche per la politica delle donne: volendo essere un movimento e non una istituzione, e cioè appoggiandosi alla vitalità delle relazioni e non a regole gerarchiche, c'è necessità di una mediazione fluida come quella dell'autorità relazionale di radice materna, piuttosto che di una formalizzazione istituzionale. $\grave{E}$ l'autorità fluida e vivente che garantisce la libertà. E d'altra parte il

\footnotetext{
16 Vedi su questo Luisa Muraro, Autorità, cit., pp. 96-101.

${ }^{17}$ Cfr. Hannah Arendt, "Che cos'è l'autorità?", in Hannah Arendt, Tra passato e futuro, tr. it. di Tania Gargiulo, Garzanti, Milano 1991, pp. 130-192.

${ }^{18}$ Cfr. ivi, p. 141.
} 
riconoscimento dell'autorità non può essere che libero, altrimenti scivola nel potere.

\section{Il conflitto asimmetrico nel simbolico}

L'aver parlato della figura simbolica dell'autorità di matrice femminile mi permette di introdurre un altro aspetto per il quale la politica è simbolica. Ripartiamo dal fatto che nel linguaggio simbolico storico che noi parliamo il movimento delle donne ha introdotto un concetto che prima non esisteva: l'autorità politica di radice materna. Sottolineo l'aggettivo "politica" perché è solo nel patriarcato che la relazione con la madre viene vista come privata ovvero non politica. Tutto questo porta con sé a cascata una serie di altri concetti innovativi riguardanti la politica, la libertà, le relazioni e così via, che lascio solo intuire. L'aver portato al centro questa figura significa un'apertura di scambio e allo stesso tempo di conflitto con altre concezioni della politica, del potere e dell'autorità. L'invenzione di una figura simbolica come questa non nasce tanto dall'impegno a trasformare il linguaggio per modificare i limiti tra ciò che è dicibile e ciò che non è dicibile, nel senso espresso da Michel Foucault. In altre parole lo scambio e il conflitto politico non avvengono solo sul piano linguistico. È qui il punto in cui si coglie con maggiore chiarezza come nella politica delle donne vi sia un circolo tra linguaggio ed esperienza che invece manca nella concezione che considera il linguaggio simbolico come il livello unico di scontro politico, ritenendolo soltanto mezzo per influenzare e performare l'esperienza.

Un esempio interessante per comprendere cosa sia il conflitto nel simbolico, quando lo si interpreti come l'unico livello che ha effetti sull'esperienza soggettiva è il dibattito che si è aperto attorno alla parola "popolo" negli ultimi anni. Una battaglia simbolica che vede impegnate parti culturali e politiche profondamente diverse. Penso a Laclau e alla sua ripresa del concetto gramsciano di egemonia in relazione alla costruzione di un popolo come 
totalità aperta ${ }^{19}$. E penso però anche alla dialettica tra sovrano e popolo come emerge nella destra europea a partire da Marine Le Pen già nel 2012, per cui la sovranità nazionale si sovrappone e rimanda alla sovranità popolare e ad un Io sovrano creativo in chiave neoliberale ${ }^{20}$. Questa battaglia simbolica come anche quella con la coppia "stato-società civile" mostrano una modalità retorico-linguistica per orientare l'immaginario e l'azione dei soggetti. Nel caso di questo conflitto si combatte attorno a diverse interpretazioni della parola "popolo", perché ognuna di queste interpretazioni ha effetti politici performativi diversi all'interno di un governo della realtà.

Ho ragionato su questo esempio per fare la differenza. Infatti è vero che il movimento delle donne ha portato diversi concetti nello scambio e nel conflitto del simbolico (autorità, libertà, politica relazionale, disparità, coincidenza dei fini con i mezzi), però non è mai stato in vista di una performatività dei comportamenti e per un governo della realtà. Si è trattato di portare al linguaggio e dunque potenzialmente a tutti i parlanti quello che è stato guadagnato in pratiche di donne in tempi scanditi dalla necessità interna del processo, strutturati attorno a relazioni viventi ed affettive. Pratiche che hanno avuto la capacità di suggerire strade trasformative per le donne che avevano a cuore la libertà e che possono risultare vere e riprese anche da altre donne e da uomini che hanno il gusto di una libertà sperimentata e significata in prima persona.

Non si tratta di una posizione ingenua che sottovaluta la capacità performativa del linguaggio simbolico, ma di far andare intrecciate la critica ai significanti dominanti - quelli che costringono in ruoli definiti sia donne sia uomini - con l'invenzione di pratiche che sperimentano esercizi di libertà

${ }^{19}$ Cfr. Ernesto Laclau, "Il ruolo dell'universale nella costruzione delle logiche politiche", in Judith Butler, Ernesto Laclau, Slavoj Žižek, Dialoghi sulla sinistra. Contingenza, egemonia, universalità (2000), tr. it. di Diego Ferrante, Laterza, Bari 2010, pp. 54-63.

20 Vedi sulla saldatura tra Stato sovrano, popolo sovrano e Io sovrano e sul legame con il neocapitalismo Ida Dominijanni, "La trappola sovranista", Parole chiave, XXVIII, 2020, pp. 17 34 . 
nel concreto dei contesti vissuti. Questo doppio nesso di critica e di sperimentazione di vie trasformative mostra come il movimento delle donne non sia mai stato antagonista. Cioè non ha agito la contrapposizione pura e semplice, perché ha sempre cercato di creare situazioni in cui vivere subito nel presente forme di libertà da scoprire ed inventare, cercando le parole giuste per dire quel che si è vissuto. Inoltre l'entrare in conflitto con il simbolico dominante nasce dal desiderio di trasformare il linguaggio di tutti, perché solo così una concezione nuova diventa circolante e va oltre l'esperienza contestuale in cui è stata generata. E tuttavia fa parte della concretezza di questa politica l'invito a ricordare sempre di nuovo la genesi storica delle figure e dei concetti, in modo che sia presente a chi li adopera. Si tratta della tensione fertile tra linguaggio ed esperienza vissuta che il movimento delle donne ha praticato: qualsiasi aggiramento delle radici storiche dell'esperienza renderebbe un concetto solo un segno linguistico strumentalizzabile e non una forma simbolica vivente. Dunque non c'è niente di arbitrario e strumentale in questa concezione del linguaggio.

Penso che per capire al meglio la politica simbolica del femminismo sia rileggere il testo di Hannah Arendt Teoria del giudizio politico, in cui Arendt riprende in chiave politica il giudizio di gusto di Kant espresso nella Critica del giudizio ${ }^{21}$. Dove il gusto per Arendt è un giudizio sensibile singolare che porta a discriminare il giusto e lo sbagliato sulla base del sentir piacere o dispiacere, ovvero in base appunto al senso del gusto ${ }^{22}$. Il giudizio singolare non è universale in senso logico, ma pretende l'universalità, e cioè l'accordo degli altri. In questo senso è politico, in quanto presuppone nello spazio

\footnotetext{
${ }^{21}$ Ricordiamo in particolare i paragrafi 31-35 del libro II di Immanuel Kant, Critica del giudizio (1790), tr. it. di Alfredo Gargiulo, Laterza, Bari 1992, pp. 109-114.

${ }^{22}$ Cfr. Hannah Arendt, Teoria del giudizio politico, tr. it. di Pier Paolo Portinaro, il melangolo, Genova 1990, in particolare pp. 97-99.
} 
pubblico una risposta da parte degli altri, sollecitandoli, piuttosto che dare per scontata una comunità politica di appartenenza ${ }^{23}$.

Considero questa concezione di Arendt rispondente a come il movimento delle donne si è mosso sul piano simbolico ${ }^{24}$. Trovare le parole per dire l'esperienza singolare ha significato fare una scommessa di verità che viene proposta come condivisibile anche da altri secondo una tensione verso l'universalità, che però poi non viene condivisa di fatto. È una scommessa di giudizio comune, e cioè che vi sia accordo da parte degli altri riguardo a quella verità guadagnata nell'esperienza, che gli altri non hanno vissuto. In quanto scommessa, può riuscire ma anche fallire.

In questo contesto è il concetto di esperienza a trasformarsi. Non si tratta di qualcosa di semplicemente soggettivo. Certo si vive un'esperienza in prima persona, ma esprimerne la verità e mostrarne l'importanza per altri mostra come l'esperienza sia più che personale. È politica in senso ampio, dando per presupposto uno scambio nel simbolico.

\footnotetext{
${ }^{23}$ Cfr. ivi, p. 103. Su questa interpretazione che Arendt dà del giudizio estetico kantiano si legga Edoardo Greblo, "Il poeta cieco. Hannah Arendt e il giudizio", aut aut, CCXXXIX-CCCXL, 1990, pp. 111-126, che insiste sull'analogia tra giudizio politico e giudizio estetico nel pensiero di Arendt. Su questo si veda anche Laura Boella, Il coraggio dell'etica. Per una nuova immaginazione morale, Cortina, Milano 2012, pp. 94-102. Si veda anche Stefania Tarantino, Chiaroscuri della ragione. Kant e le filosofe del Novecento, Guida, Napoli 2018.

${ }^{24}$ Sappiamo che Hannah Arendt non si è definita femminista. Tuttavia, nell'intervista a Günther Gaus del 1964, Arendt, rispondendo alla domanda se la questione dell'emancipazione fosse importante per lei, parla di qualità femminili che vanno salvaguardate, e che a questo si è attenuta nella sua vita. Vedi Hannah Arendt, "Che cosa resta? Resta la lingua materna. Conversazione di Hannah Arendt con Günter Gaus", aut aut, CCXXXIX-CCXL, 1990, pp. 11-30, qui p. 12. Adriana Cavarero ha scritto per dire in che senso il pensiero di Arendt abbia una vicinanza con le interrogazioni poste dal pensiero della differenza sessuale, così che è soprattutto in questa chiave che le categorie di Arendt possono essere riprese e valutate. Si veda Adriana Cavarero, "Dire la nascita", in Diotima, Mettere al mondo il mondo. Oggetto e oggettività alla luce della differenza sessuale, La Tartaruga, Milano 1990, pp. 95-99. Sulle letture femministe di Arendt si legga Margarete Durst, "Hannah Arendt e le interpretazioni femministe e al femminile del suo pensiero. Una circolarità virtuosa", Annali di studi religiosi, v, 2004, pp. 295-308.
} 


\section{Per una conclusione}

Quello che ho cercato di far vedere in questo testo è che il pensiero femminista è partito dalla creazione di forme di vita libere, processuali e in fieri. Radicato in esse, ha espresso prese di posizione, affermazioni sensate, enunciati veri nel linguaggio simbolico di tutti. Queste forme concrete di esistenza - le pratiche che divengono forme di vita - sono nate da esperienze discusse e agite con altre per il desiderio di vivere subito, senza procrastinare, legami politici vincolanti tra donne, realizzando stili nuovi sia sul piano materiale che culturale.

È questo il punto focale: non si è entrate nel conflitto del simbolico portando una posizione solo ragionata, bensì una posizione che ha avuto radici in forme di vita sperimentate e sperimentali. Per questo va considerato fondante e logicamente primo il circolo tra pensiero pensante ed esperienza vissuta con altre. Questo però comporta, in ogni momento, di saper declinare i significati già a disposizione nel linguaggio comune e renderli duttili per portarli a significare altro. Nella consapevolezza che il conflitto sulle parole e sugli enunciati ha a che fare con la performatività linguistica, ma senza avere questo effetto come prima intenzione.

Il secondo punto che il testo mette in evidenza è che nelle stesse pratiche femministe vi sono elementi simbolici portanti. Questo è stato illustrato negli esempi presentati: l'agire l'autocoscienza e l'autorità. Ma si poteva estendere questa analisi anche ad altre pratiche oggi altrettanto vitali. Il fatto è che il simbolico fa fare salti di realtà e permette di collegare parti impreviste d'esperienza. Lo abbiamo illustrato in rapporto all'après-coup come lo si può vedere rispetto alla libertà relazionale nell'autorità.

Il terzo punto, che coinvolge il simbolico, è la scommessa politica che viene messa in campo ogni volta che si ha un guadagno di verità a partire dalle pratiche vissute in prima persona nella relazione con altre. Al centro è l'idea che ciò che si coglie di vero nell'esperienza contingente può essere offerto nello 
scambio linguistico a tutti, dove è fondamentale come venga recepito e ripreso. Questo in sintonia con l'interpretazione arendtiana del giudizio di gusto, che pretende l'assenso o il dissenso altrui, creando così legami politici imprevisti. È per questo che si tratta di una scommessa simbolica, in quanto noi non possiamo prevedere la risposta che altri possono dare al guadagno di verità che noi portiamo.

\section{Nota bibliografica}

ARENDT, Hannah, "Che cos'è l'autorità?", in Hannah Arendt, Tra passato e futuro (1961), tr. it. di Tania Gargiulo, Garzanti, Milano 1991, pp. 130-192.

—, Teoria del giudizio politico (1982), tr. it. di Pier Paolo Portinaro, il melangolo, Genova 1990.

—, "Che cosa resta? Resta la lingua materna. Conversazione di Hannah Arendt con Günter Gaus", aut aut, CCXXXIX-CCXL, 1990, pp. 11-30.

BERGER, Anne Emmanuelle, And Yet she speaks! "Italian feminism" and Language, in Casarino Cesare, Righi Andrea (ed. by), Another Mother: Diotima and the Symbolic Order of Italian Feminism, University of Minnesota Press, Minneapolis 2018.

BOELLA, Laura, Il coraggio dell'etica. Per una nuova immaginazione morale, Cortina, Milano 2012.

BERNINI, Lorenzo, Le pecore e il pastore. Critica, politica, etica nel pensiero di Michel Foucault, Liguori, Napoli 2008.

BERTILOTTI, Teresa, SCATTIGNO, Anna, Il femminismo degli anni Settanta, Viella, Roma 2005. 
CANNAMELA, Danila, “I am an atypical mother': Motherhood and maternal language in Giovanna Cristina Vivinetto's poetry”, Forum Italicum, LV, 2021, pp. pp. 85-108.

CAVARERO, Adriana, Nonostante Platone, Editori Riuniti, Roma 1990.

—, "Dire la nascita", in Diotima, Mettere al mondo il mondo. Oggetto e oggettività alla luce della differenza sessuale, La Tartaruga, Milano 1990, pp. 95-99.

—, Tu che mi guardi, tu che mi racconti, Feltrinelli, Milano 1997.

CIGARINI, Lia, La politica del desiderio, Pratiche Editrice, Parma 1995.

CIMATTI, Felice, Il taglio. Linguaggio e pulsioni di morte, Quodlibet, Macerata 2015.

DURST, Margherita, "Hannah Arendt e le interpretazioni femministe e al femminile del suo pensiero. Una circolarità virtuosa”, Annali di studi religiosi, v, 2004, pp. 295-308.

DELEUZE, Gilles, Il sapere. Corso su Michel Foucault (1985-1986), vol. I, tr. it. di Lorenzo Fantin, Ombre Corte, Verona 2014.

—, Foucault (1986), tr. it. di Filippo Domenicali, Orthotes, Napoli 2018.

DINI, Tristana, La materiale vita. Biopolitica, vita sacra, differenza sessuale, Mimesis, Milano 2016.

DIOTIMA, Il pensiero della differenza sessuale, La Tartaruga, Milano 1987.

—, Oltre l'uguaglianza. Le radici femminili dell'autorità, Liguori, Napoli 1995.

—, Femminismo fuori sesto. Un movimento che non può fermarsi, Liguori, Napoli 2017. 
DOMINIJANNI, Ida, "Politica del simbolico e mutamento", IG Informazioni, III, 1992, pp. 131-157.

—, "La trappola sovranista", Parole chiave, XXVIII, 2020, pp. 17-34.

DUROUX, Françoise, Il paradigma perturbante della differenza sessuale. Una filosofia femminista, tr. it. a cura di Stefania Tarantino e Chiara Zamboni, Mimesis, Milano 2021.

FORCINA, Marisa, Rappresentazioni politiche della differenza, FrancoAngeli, Milano 2009.

FOUCAULT, Michel, L'ordine del discorso (1971), tr. it. di Alessandro Fontana, Einaudi, Torino 1985.

FRAIRE, Manuela, "Attualità e inattualità dell'autocoscienza”, in Chiara Zamboni (a cura di), La carta coperta. L’inconscio nelle pratiche femministe, Moretti\&Vitali, Bergamo 2019, pp. 54-60.

FRAISSE, Geneviève, Il mondo è sessuato. Femminismo e altre sovversioni (2016), tr. it. a cura di Annarosa Buttarelli, Nottetempo, Milano 2019.

GREBLO, Edoardo, "Il poeta cieco. Hannah Arendt e il giudizio", aut aut, CCXXXIX-CCXL, 1990, pp. 111-126.

GUARALDO, Olivia, Politica e racconto. Trame arendtiane della modernità, Meltemi, Roma 2003.

KANT, Immanuel, Critica del giudizio (1790), tr. it. di Alfredo Gargiulo, Laterza, Bari 1992.

LACAN, Jacques, La psicosi. Seminario III, tr. it. a cura di Giacomo Contri, Einaudi, Torino 1985. 
LACLAU, Ernesto, "Il ruolo dell'universale nella costruzione delle logiche politiche”, in Judith Butler, Ernesto Laclau, Slavoj Žižek, Dialoghi sulla sinistra. Contingenza, egemonia, universalità (2000), tr. it. di Diego Ferrante, Laterza, Bari 2010.

LONZI, Carla, Taci, anzi parla. Diario di una femminista, ed. Rivolta femminile, Milano 1978.

LIBRERIA DELLE DONNE DI MILANO, Non credere di avere dei diritti. La generazione della libertà femminile nell'idea e nelle vicende di un gruppo di donne, Rosenberg\&Sellier, Torino 1989.

MUNI, Andrea, "Foucault e Lacan. L'amicizia, il discorso, il soggetto etico", aut aut, CCCLXXXVII, 2020, pp. 69-86.

MURARO, Luisa, L'ordine simbolico della madre, Editori Riuniti, Roma 1991.

—, Autorità, Rosenberg\&Sellier, Torino 2013.

RISOLDI, Maria Chiara, \#Me Too: il patriarcato dalle mimose all'hashtag, Antonio Trombolini Editore, Loreto 2018.

SCARAMUZZA, Gabriele, Per Dino Formaggio, in Gabriele Scaramuzza, Smarrimento e scrittura, Mimesis, Milano 2019.

RECALCATI, Massimo, Jacques Lacan. Desiderio, godimento e soggettivazione, Cortina, Milano 2012.

—, L’ora di lezione. Per un'erotica dell'insegnamento, Einaudi, Torino 2014.

SANTINI, Marina, TAVERNINI, Luciana, Mia madre femminista. Voci da una rivoluzione che continua, Il Poligrafo, Padova 2015.

TARANTINO, Stefania, Kant e le filosofe del Novecento, Guida, Napoli 2018. 
VACCARO, Salvo, "Lo sguardo e la differenza. Michel Foucault e il pensiero delle donne", in Salvo Vaccaro e Mario Coglitore (a cura di), Michel Foucault e il divenire donna, Mimesis, Milano 1997, pp. 17-45.

ZERILLI, Linda, Femminism and the Abyss of Freedom, University of Chicago Press, Chicago 2005.

ZUPANČIČ, Alenka, Che cos'è il sesso? (2017), tr. it. di Pietro Bianchi, Ponte alle Grazie, Milano 2018.

\section{Nota biografica}

Chiara Zamboni insegna Filosofia teoretica all'Università di Verona. Da anni si occupa di pensiero delle donne e ha dato vita con altre alla comunità filosofica Diotima. Tra le sue pubblicazioni: Parole non consumate. Donne e uomini nel linguaggio (Liguori, Napoli 2001), Pensare in presenza. Conversazioni, luoghi, improvvisazioni (Liguori, Napoli 2009), Sentire e scrivere la natura (Mimesis, Milano-Udine 2020). Ha curato María Zambrano. In fedeltà alla parola vivente (Alinea, Firenze 2002), Il cuore sacro della lingua (Il Poligrafo, Padova 2006), L’inconscio può pensare? Tra filosofia e psicoanalisi (Moretti\&Vitali, Bergamo 2013), Una filosofia femminista. In dialogo con Françoise Collin (Manni, Lecce 2015), La carta coperta. L’inconscio nelle pratiche femministe (Moretti\&Vitali, Bergamo 2019). Ha collaborato ai diversi volumi di Diotima, pubblicati dal 1987 ad oggi. Da anni è docente nel Master di Duoda. Estudios de la Diferencia Sexual (Università di Barcellona). 